Forum Kind Jugend Sport 2021 $\cdot$ 2:158-163 https://doi.org/10.1007/s43594-021-00049-2 Angenommen: 8. Oktober 2021 Online publiziert: 8. November 2021 ๑ Der/die Autor(en) 2021

\title{
Juliane Lanz
}

Institut für Sonderpädagogische Entwicklungsförderung und Rehabilitation, Arbeitsstelle Pädagogische Lesungen, Universität Rostock, Rostock, Deutschland

\section{Bouldern hat Potenzial}

\section{Eine Standortbestimmung für Kinder und Jugendliche nach der Corona-Pandemie}

Herausforderungen, aber auch Möglichkeiten. wie der Sport, sowohl bei den kommerziellen Anbietern als auch in den Vereinen, zu den Verlierern der CoronaPandemie (Walper et al. 2021; DOSB 2020). An der Schnittstelle, dem Kinder- und Jugendsport, gilt es, Strukturen zu (re-)etablieren, um diese Lücke etwas zu schließen. Ansprechende Angebote könnten sich beispielsweise aus den neuen olympischen Sportarten ergeben, die in Tokio 2021 Premiere hatten. Neben Baseball, Skateboarding, Karate und Surfen war erstmals auch das Sportklettern bei Olympischen Spielen vertreten. In einem Dreikampfaus Speedklettern (Schnellklettern auf einer weltweit einheitlichen Route), Leadklettern (am Seil an stets neuen Routen) und Bouldern wurden die weltbesten Athlet*innen des Klettersports gekürt. Bouldern ist im Kontext der Gewinnung neuer Sportler*innen von besonderem Interesse, wenn auch nicht ohne Schwierigkeiten:

- Durch die häufige Bindung des Sports an kommerzielle Sportanbieter, seltener jedoch an den Vereinssport, steht Bouldern bisher nicht vielen Kindern und Jugendlichen offen.

- Durch die ihm innewohnenden Potenziale bietet Bouldern vor allem Jugendlichen breite Möglichkeiten und Entwicklungsperspektiven.

- Für eine noch ,junge" Sportart (die sich erst in den vergangenen zehn Jahren etabliert hat) mit wenig Bindung und Verankerung im gemeinnützigen, organisierten Jugendsport ergeben sich besondere
Es ist Ziel der vorliegenden Darstellung, den Bouldersport vorzustellen und in der Sportlandschaft zu verorten, um die damit verbundenen Möglichkeiten für Kinder und vor allem Jugendliche aufzuzeigen. Daraus ergeben sich in der Folge Anregungen für Entscheidungsträger*innen im Sport, attraktive und inkludierende Angebote zu schaffen.

\section{Bouldern in Deutschland}

Beim Bouldern klettert man an senkrechten und geneigten Felswänden. In Hallen geschieht dies an Kunststoffgriffen (• Abb. 1). Im Unterschied zum Sportklettern sind die Wände mit maximal vier bis fünf Metern deutlich weniger hoch, weswegen auf eine Seilsicherung verzichtet wird. Zur Verletzungsprävention ist der Boden mit dicken Matten ausgelegt. Farbig markierte Routen zeigen unterschiedliche Schwierigkeitsgrade.

Da Bouldern als Sport in Deutschland recht neu und teilweise noch nicht etabliert ist, fehlen an vielen Standorten die Strukturen, die man von anderen Sportarten kennt. Unter den knapp 10.000 kommerziellen Sportanbietern in Deutschland befinden sich knapp 500 Kletterhallen, davon ein Drittel zum Bouldern ausgelegt, von denen 200, dann nicht-kommerziell, vom deutschen Alpenverein betrieben werden (DAV 2018). In einigen Fällen ist Bouldern ein Angebot einer Kletterhalle, die zusätzlich ein Boulderareal anbietet. Letztere werden in Deutschland teilweise in Kooperation mit dem Deutschen Alpenverein (DAV) betrieben. Viele Kletterhallen, die nicht zum DAV gehören, sind im Branchenverband Kletterhallenverband (KLEVER) e.V. organisiert, die meisten Boulderhallen jedoch nicht. Der Verband weist 53 Mitglieder aus, 13 davon sind reine Boulderhallen (Kletterhallenverband (KLEVER) e. V. 2021).

Die Mehrheit der Hallen wurde in den vergangenen fünf bis zehn Jahren gegründet. ${ }^{1}$ Die Anzahl der Mitglieder in den einzelnen Sportstätten ist sehr unterschiedlich, da diese auf verschiedene Weise gezählt werden. In einigen Fällen werden alle Personen eingerechnet, die jemals in der entsprechenden Halle bouldern waren. Andere Einrichtungen erfassen Personen erst bei mehrfachen Besuchen, wieder andere Boulderhallen dokumentieren nur Sportler*innen, die regelmäßig vor Ort sind. Zur Orientierung sei angegeben, dass die Mehrzahl der Hallen zwischen 7000 und 50.000 Personen angibt, wobei die Anzahl der Mitglieder und das Bestehen der Halle nur bedingt im Verhältnis stehen. Vielmehr steht die Mitgliederzahl in Abhängigkeit des Ortes, an dem sich die Boulderhalle befindet. In Ballungsräumen und Universitätsstädten scheint der Zulauf verhältnismäßig höher.

Ähnlich der Betätigung in einem Fitnessstudio beschäftigen sich Sportler*innen in Boulderhallen selbstständig, setzen sich Trainingsziele und verfolgen diese. Da die körperliche Anstrengung enorm ist, bleibt ein großer Teil der Aufenthaltszeit in einer Boulderhalle

\footnotetext{
1 Die Verfasserin sprach mit 16 Betreiber*innen von Boulderhallen und erhielt von innen konkrete Daten zu ihrem Betrieb.
} 


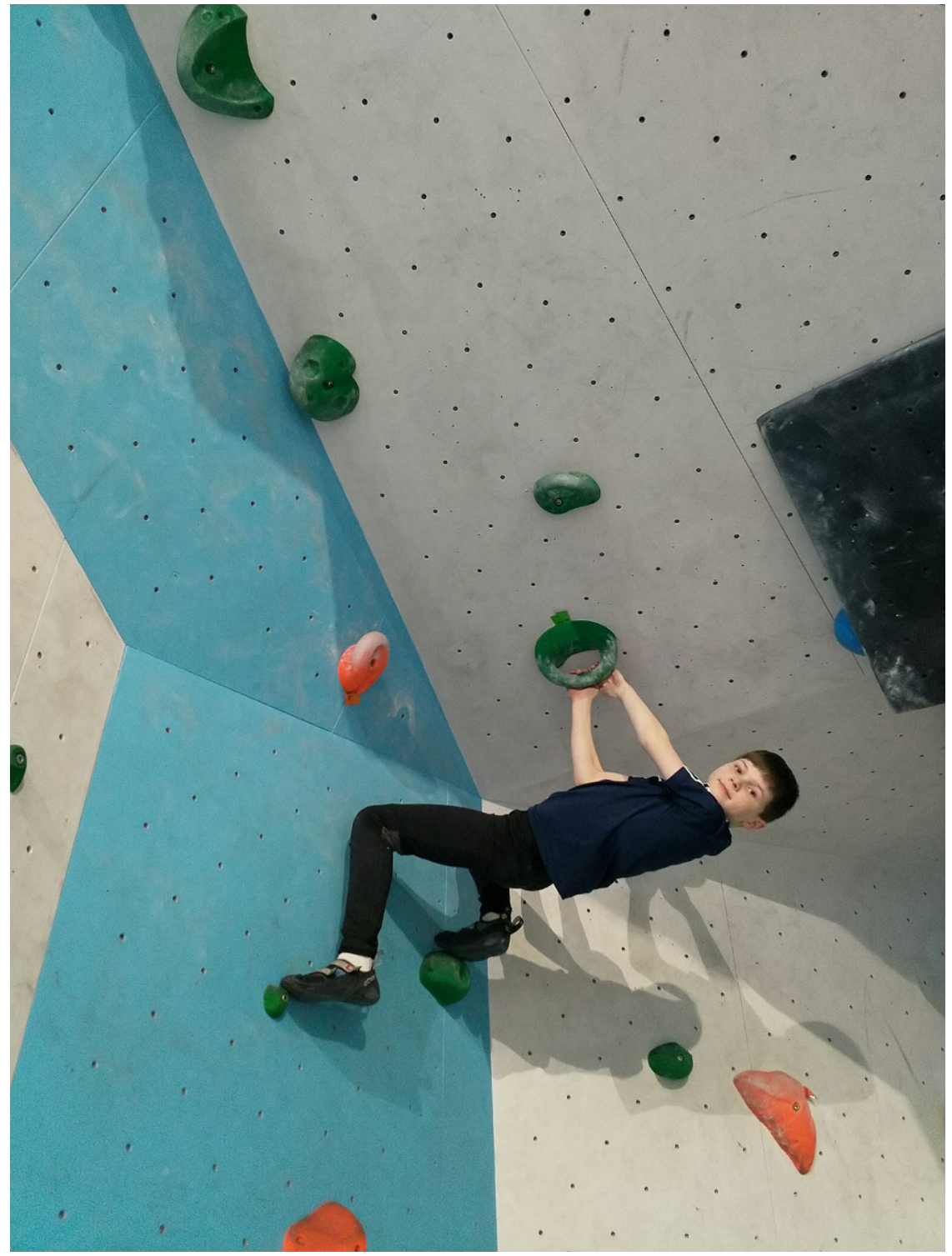

Abb. $1 \Delta$ Beim Bouldern klettert man an senkrechten und geneigten Felswänden. In Hallen geschieht dies an Kunststoffgriffen. (Foto: Juliane Lanz)

den Pausen vorbehalten. Diese dienen der kurzfristigen Regeneration, der Diskussion von Aufstiegsstrategien mit anderen Bouldernden und der psychischen Vorbereitung auf die Bewältigung der nächsten Route. Diese sehr individuelle Beschäftigung ist im Kinder- und Jugendbereich wenig ausgeprägt. Nur ältere Jugendliche (oftmals ab 14 oder 16 Jahren) dürfen ohne Aufsichtsperson eine Boulderhalle besuchen, es sei denn, sie nehmen an einem organisierten Kurs teil. Viele Minderjährige kommen ausschließlich mit ihren ebenfalls bouldernden Eltern in die Hallen. Insgesamt haben die meisten Betreiber*innen
Die Finanzierungsmodelle sind in allen Hallen sehr ähnlich. Neben Monats- oder Jahreskarten werden den Kund ${ }^{\star}$ innen oft Tagestickets, sogenannte Elferkarten (elfmal bouldern, zehnmal bezahlen) oder Familienkarten angeboten. An vielen Standorten haben auch Kinder und Jugendliche, außer vielleicht einer Ermäßigung, keine anderen Optionen als die genannten. Damit kann ein Besuch einer Boulderhalle rasch kostspielig werden und übersteigt mit einem durchschnittlichen Eintritt von fünf bis acht Euro die frei verfügbaren finanziellen Spielräume vieler Jugendlicher (Langmeyer und Winklhofer 2014). Deswegen spielen meist Eltern oder andere nahestehende Erwachsene eine große Rolle für den Zugang zu diesem Sport. Aufgrund der fehlenden Vereinsstrukturen und dem geringen Organisationsgrad in Branchenverbänden ist die Zahl der aktiven Bouldersportlerinnen weder für den Bereich der Kinder und Jugendlichen noch der Erwachsenen in Deutschland zu ermitteln.

Wettkampfsportlich findet eine Reihe freier und regionaler Veranstaltungen statt. Auf leistungssportlichem Niveau sind Bouldern und Klettern eng verknüpft. Als sportlicher Dachverband agiert der DAV. Dessen Organisation über die regionalen Sektionen ist sehr heterogen und wirkt nur punktuell auf den Bouldersport. Mit der Zulassung zu den Olympischen Spielen wird der Leistungssport durch den DAV und das dort angebundene Trainer*innenteam koordiniert. Als Weltsportverband fungiert die International Federation of Sports Climbing (IFSC).

Boulderhallen angegeben, dass etwa 10 bis $30 \%$ der Nutzer ${ }^{\star}$ innen Kinder und Jugendliche seien. Hauptsächlich kommen diese über Schnupperkurse, Schulausflüge oder individuelle Besuche mit Aufsichtspersonen in die Hallen. Wenige nehmen an längerfristigen strukturierten Angeboten wie Trainingsgruppen, Schulsportkooperationen oder Kinderkursen teil. In einigen Fällen kooperieren die Boulderhallen mit den Sektionen des Deutschen Alpenvereins oder anderen Sportvereinen, die dann eigene Kinderund Jugendgruppen unterhalten und die Hallen über Mietverträge mitnutzen.

\section{Die Eignung für junge Menschen}

Ausgehend von der Annahme, dass viele junge Menschen ab dem Teenager ${ }^{*}$ innen-Alter ihren angestammten (Vereins-)Sport verlassen und sich anderen Formen der körperlichen Betätigung zu- oder auch vom Sport abwenden (Burrmann und Mutz 2017, S. 392), könnte diese Lücke durch neue Angebote im sportlichen Lebenslauf geschlossen oder verkleinert werden. Die Entwicklungsmöglichkeiten, aber auch die in dieser Lebensphase wahrgenommenen 


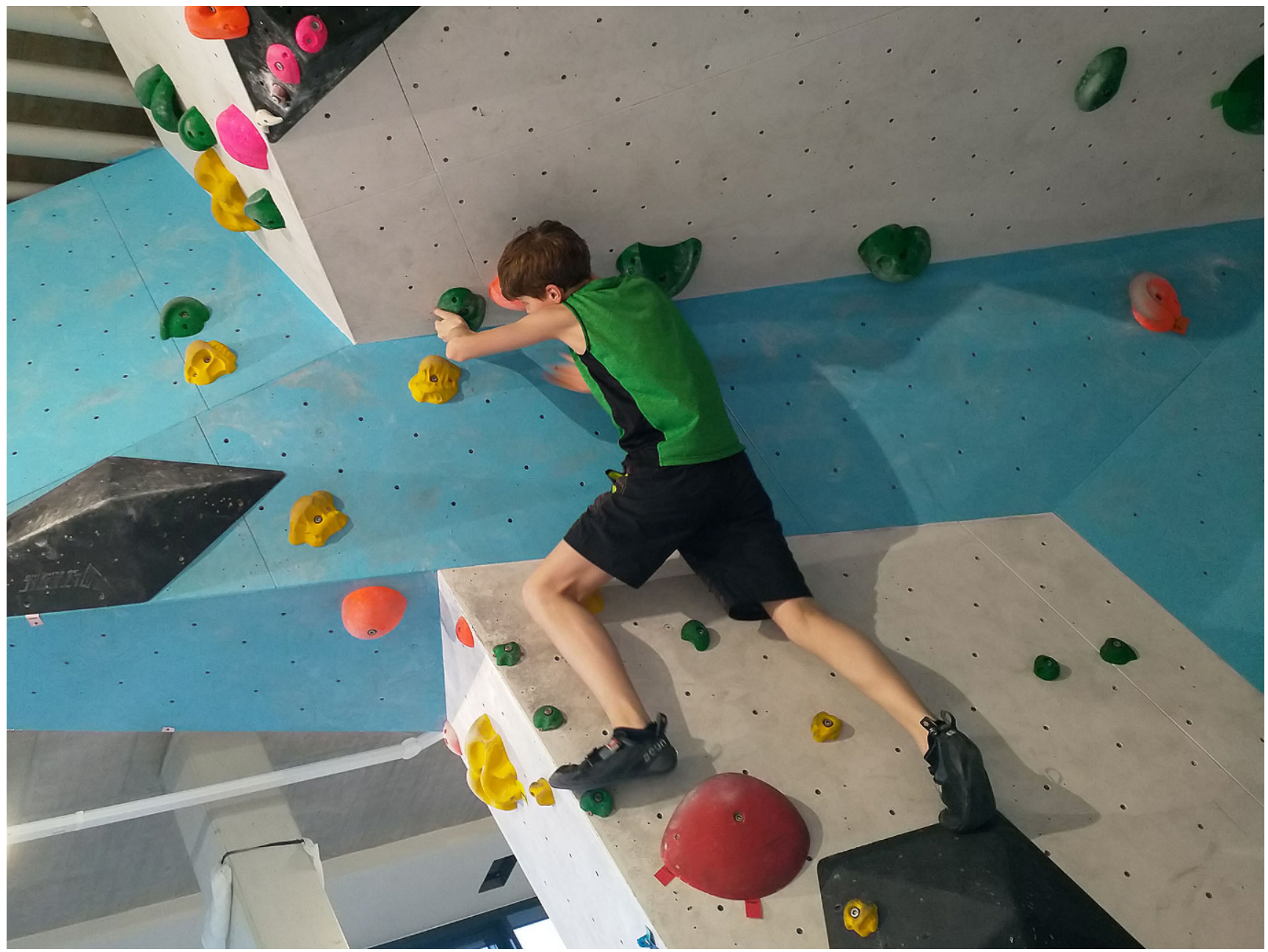

Abb. 2 \ Der Einstieg ist auf jedem Niveau möglich. (Foto: Juliane Lanz)

Einschränkungen wirken lange nach und beeinflussen die Ressourcen für die weitere Lebensführung, Bildung und sozialen Chancen (Eikenbusch 2019, S. 17). Die Ausgangssituation ist heterogen: Geschlecht, ethnische Herkunft und Angebote in der Umgebung beeinflussen auch die körperliche Aktivität und die Einstellungen zum Sport (Burrmann und Mutz 2017, S. 397). Zwanzig Prozent der Kinder in Deutschland machen konkrete Armutserfahrungen, wobei die Aussicht auf soziale Mobilität sehr gering ist (Eikenbusch 2019, S. 41). Diese Ausgangssituation verschlechtert den Zugang zu Freizeitmöglichkeiten und Sport und bleibt bei den weiteren Überlegungen vor allem deswegen im Blick, da sich Bouldern aktuell als eher exklusive Sportart darstellt, bei der - vor allem an den Standorten, an denen Schul- und/oder Vereinskooperationen fehlen - allein die materielle Situation Zugang ermöglicht.

Der Gestaltung ihrer Freizeit messen Jugendliche eine hohe Bedeutung bei. Die Möglichkeiten werden dabei immer vielfältiger. Die Hälfte aller Jugendlichen verfügt über mehr als vier Stunden Freizeit pro Tag und nutzt diese gerne hybrid, indem sie verschiedene Aktivitäten kombiniert (Eikenbusch 2019, S. 108). Beim Bouldern kommt das Zusammensein mit Mitgliedern der Peergroup zum eigentlichen Sporttreiben hinzu. Herausfordernd in der Jugendphase ist unter anderem die Notwendigkeit eines neuen Selbst- und Körperkonzepts, das zur Identitätsbildung beiträgt (Konowalczyk 2016, S. 39). Mit Veränderung der Proportionen sind neue Körpererfahrungen wichtig, wobei die Leistung nicht immer im Vordergrund stehen muss. Beim Bouldern sind Körperspannung und
Koordination in Kombination mit Geduld und Konzentration gefragt. Dazu kommt, dass ein Einstieg und Erfolgserlebnisse auf jedem Niveau möglich sind (- Abb. 2).

Aktivitäten von Jugendlichen zeichnen sich oft durch die Suche nach Aufmerksamkeit und Anerkennung und das Austesten körperlicher und psychischer Grenzen aus (Eikenbusch 2019, S. 110). Nicht nur das Testen der Grenzen, sondern auch deren Verschiebung lässt sich beim Bouldersport gut nachvollziehbar wahrnehmen. Kommt man in der einen Woche noch nicht an den Top-Griff (Ziel) einer bestimmten Route, ist dies beim nächsten oder übernächsten Mal möglich. Es gilt unter anderem Höhenangst zu überwinden und das Vertrauen in eigene Fähigkeiten aufzubauen. Rückmeldungen bei falscher Einschätzung der eigenen Fähigkeiten folgen unmittelbar (zum Bei- 


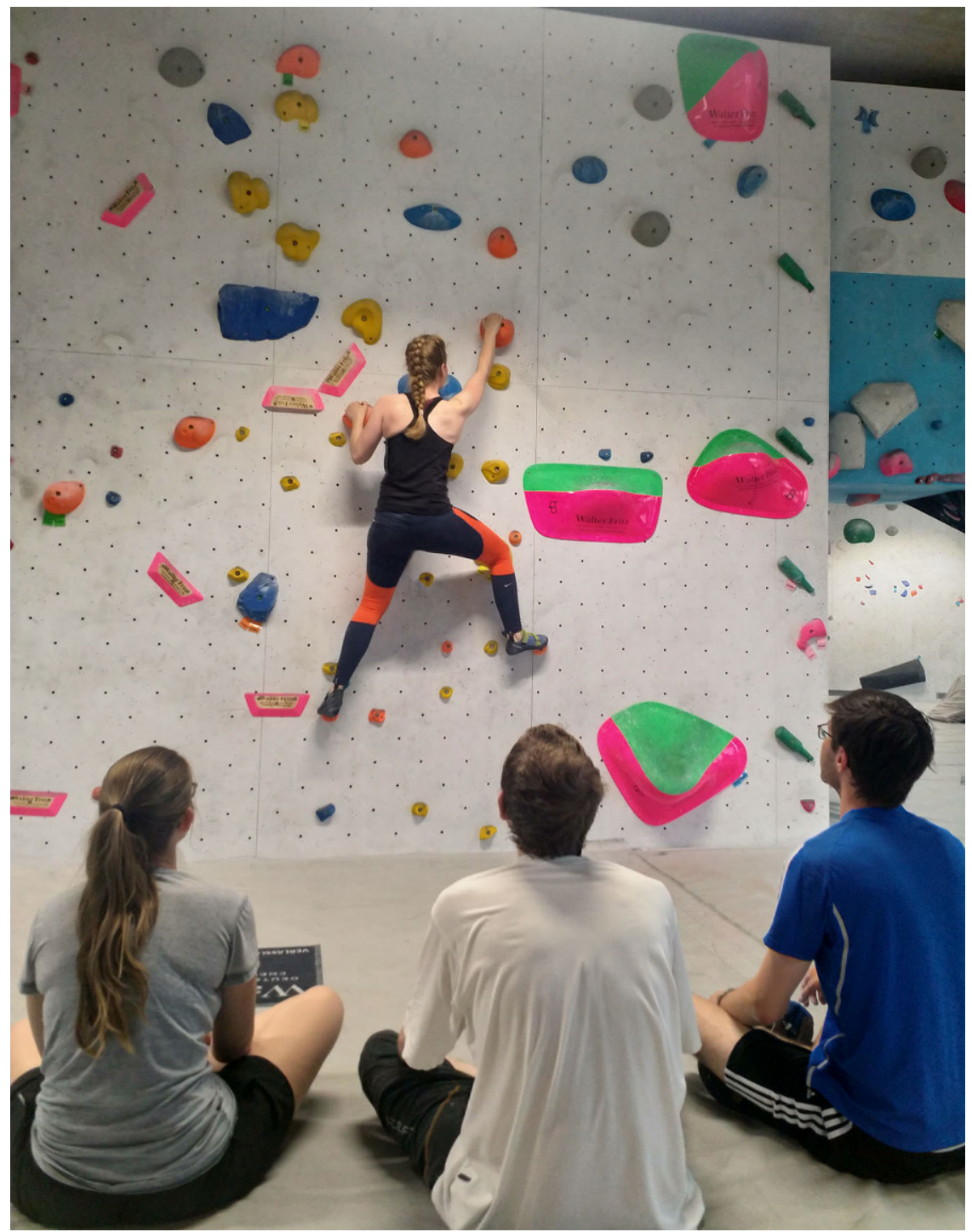

Abb. 3 A Der Wechsel zwischen Beobachtungsposition und aktiver Rolle bietet Phasen der Kommunikation und der physischen Aktivität. (Foto: Juliane Lanz)

spiel durch das Abfallen von der Wand oder das Nicht-Erreichen des Ziels) aber mit überschaubaren Konsequenzen und in der Regel großer Motivation für einen nächsten Versuch. Inwiefern Aufmerksamkeit und Anerkennung wahrgenommen werden können, liegt zum einem am Wohlwollen der Peergroup, aber auch am Geschick der Anleitenden.

Dem Konzept der Selbstentfaltung kommt eine hohe Bedeutung zu. Es lässt sich im selbstorganisierten Sport am ehesten verfolgen (Neuber 2007). Ein gutes Angebot für Jugendliche im Bouldersport wäre demnach durch einen hohen Grad an Freiheit und Selbstständigkeit gekennzeichnet (wie es auch im Erwachsenenbereich der Normalfall ist). anderen, sondern die eigene Entwicklung und Verbesserung stehen im Vordergrund.

Nicht zuletzt ermöglicht der Bouldersport eine Reihe informeller Lernsettings, die nicht intendiert sind und den Jugendlichen viel Entwicklungspotenzial bieten (Neuber 2011, S. 155). Was für den Einzelnen dabei herauskommt, ist unterschiedlich und kann beim Bouldern vom Überwinden von Angst über ein besseres Verständnis physikalischer Gesetzmäßigkeiten bis hin zu besserer sozialer Integration reichen (• Abb. 3).

Einer breiteren Partizipation junger Menschen stehen die aktuellen Strukturen beim Bouldern entgegen. Sport als zivilgesellschaftlicher Akteur lässt sich, wenn überhaupt, im verband- und vereinsgesteuerten Sport identifizieren (Braun 2011, S. 117). Hier besteht, gesamtgesellschaftlich und über das Bouldern hinaus, Nachholbedarf. Eine Teilhabe für mehr Jugendliche ist also erst dann realistisch, wenn sich die Strukturen diesbezüglich geändert beziehungsweise geöffnet haben.

Sowohl der Gewinn als auch die Hürden für den Bouldersport als Angebot für Jugendliche lassen sich kurz zusammenfassen ( $\bullet$ Tab. 1). Hierbei zeigt sich, dass die Vorteile im Bouldern selbst liegen, die Hürden im organisatorisch-strukturellen Bereich.

Die Boulderhallen blieben im langen Lockdown im Winter 2020/21, der Hochsaison des Indoor-Sports fast ein halbes Jahr geschlossen. Vielen Betreiber*innen sicherten die staatlichen Hilfen das Überleben, doch der ohnehin lose Kontakt zu den Kindern und Jugendlichen riss leider oftmals ab.

Viele Betreiber*innen gaben an, dass sie die Kindergruppen oftmals frühzeitiger schließen mussten als das freie Bouldern. Die Anzahl der Personen, die pro Flächeneinheit noch zugelassen waren, machte dies notwendig. Die oftmals neuen Kinder- und Jugendgruppen hätten sich noch nicht so fest etabliert, dass sie nach den Schließungen wieder mit einem Großteil der Mitglieder starten konnten. Die vielerorts auf die Wiederöffnungen folgenden Sommerferien und die geringere Attraktivität von Indoor-Sport im Sommer kamen erschwerend hinzu. 
Tab. 1 Eignung und Hindernisse des Bouldersports als Angebot für Jugendliche

Besondere Eignung ergibt sich aus...

Derzeit bestehende Hindernisse,

die abgebaut werden müssten:

Möglichkeit der hybriden Freizeitgestaltung, die durch die hohe Pausenanzahl begünstigt wird (Sport, Freund*innen treffen, abhängen)

Kompetenzerleben

Neue Körpererfahrungen

Identitätsbildung

Austesten psychischer und physischer Grenzen

Selbstentfaltung im wenig regulierten Raum

Informelle Lernsettings

\section{Fazit}

Für den Kinder- und Jugendsport scheint Bouldern über viel Potenzial zu verfügen. Es wäre hilfreich, stünde dieser Sport einer größeren Gruppe von jungen Menschen offen. Insofern ist jede gesellschaftliche Anstrengung mit dieser Intention zu begrüßen. Der Sport selbst bietet in seinem Aufbau und seiner Struktur vor allem für Jugendliche attraktive Anreize es muss jedoch daran gearbeitet werden, mehr offene und finanzunabhängige Angebote zu etablieren. Dort, wo bereits Kooperationen mit Sportvereinen bestehen, scheint dies besser zu funktionieren. Daneben gilt es generell, die Frage des Zugangs zu klären, denn, ohne „Anlass" werden die wenigsten Jugendlichen eigenständig eine Boulderhalle ansteuern. Internationale Vorbildwirkung für die Integration aller Interessierter in den Klettersport hat die Boulder- und Kletterhalle Memphis Rox in den USA. Getragen durch die Non-Profit-Organisation One Family Memphis lautet das Motto der 2018 eröffneten modernen Boulder- und Kletterhalle: „We exclude no one - regardless of ability to pay“ (Memphis Rox 2021). Damit steht der Kletterund Bouldersport Kindern und Jugendlichen aus allen gesellschaftlichen Schichten offen und wird finanziell vor allem durch Spenden und die Mitgliedsbeiträge der zahlungskräftigeren Klientel getragen. Das Beispiel zeigt, dass es wichtig wäre, Bouldern nicht nur als „Produkt" $\mathrm{zu}$ bewerben, sondern offene und inkludierende Angebote zu unterbreiten. Diese Änderung geschieht jedoch nicht von selbst, sondern benötigt interessierte und engagierte Personen - beispielswei- se in Mehrspartenvereinen, kommunalen Sportämtern oder den Sektionen des Alpenvereins. Die bestehenden Boulderhallen müssen in so einem Prozess ihre wirtschaftlichen Interessen gewahrt sehen und würden diesen dann produktiv begleiten können. Viele Betreiber*innen von Boulderhallen haben angegeben, ihr Geschäft „nicht um reich zu werden“ $\mathrm{zu}$ betreiben, sondern um sportliche Werte $\mathrm{zu}$ vermitteln. Eine gewisse Offenheit gegenüber Pilotprojekten wäre also zu erwarten. Die durch die Olympischen Spiele erzeugte Aufmerksamkeit ließe sich sicherlich nutzen.

\section{Korrespondenzadresse}

\section{Dr. Juliane Lanz}

Institut für Sonderpädagogische Entwicklungsförderung und Rehabilitation, Arbeitsstelle Pädagogische Lesungen, Universität Rostock Rostock, Deutschland

juliane.lanz@uni-rostock.de

Danksagung. Teile der Recherche und der Gespräche lagen in den Händen der studentischen Hilfskraft Kristine Stöcker. Für diese umsichtige Unterstützung möchte ich mich herzlich bedanken und darauf verweisen, dass ich Inhalt und Schlussfolgerungen allein verantworte.

Funding. Open Access funding enabled and organized by Projekt DEAL.

Open Access. Dieser Artikel wird unter der Creative Commons Namensnennung 4.0 International Lizenz veröffentlicht, welche die Nutzung, Vervielfältigung, Bearbeitung, Verbreitung und Wiedergabe in jeglichem Medium und Format erlaubt, sofern Sie den/die ursprünglichen Autor(en) und die Quelle ordnungsgemäß nennen, einen Link zur Creative Commons Lizenz beifügen und angeben, ob Änderungen vorgenommen wurden.

Die in diesem Artikel enthaltenen Bilder und sonstiges Drittmaterial unterliegen ebenfalls der genannten Creative Commons Lizenz, sofern sich aus der Abbil- dungslegende nichts anderes ergibt. Sofern das betreffende Material nicht unter der genannten Creative Commons Lizenz steht und die betreffende Handlung nicht nach gesetzlichen Vorschriften erlaubt ist, ist für die oben aufgeführten Weiterverwendungen des Materials die Einwilligung des jeweiligen Rechteinhabers einzuholen.

Weitere Details zur Lizenz entnehmen Sie bitte der Lizenzinformation auf http://creativecommons.org/ licenses/by/4.0/deed.de.

\section{Literatur}

Braun, S. (2011). Bildungspotenziale der Zivilgesellschaft - gesellschaftspolitische Perspektiven einer bildungsbezogenen Engagementpolitik von Sportverbänden und -vereinen. In M. Krüger \& N. Neuber (Hrsg.), Bildung im Sport. Beiträge zu einer zeitgemäßen Bildungsdebatte 1. Aufl. Bildung und Sport, (Bd. 1, S. 105-120). Wiesbaden: VS. https://doi.org/10.1007/978-3-531-940267.

Burrmann, U., \& Mutz, M. (2017). Sport- und Bewegungsaktivitäten von Jugendlichen in Deutschland: ein aktueller Überblick im Spannungsfeld von "Versportung" und "Bewegungsmangel". Diskurs Kindheits- und Jugendforschung / Discourse. Journal of Childhood and Adolescence Research, 12(4), 385-401. https://doi.org/10. 3224/diskurs.v12i4.01.

DAV (2018). Klettern in Deutschland - Zahlen, Daten \& Fakten. München. https://www.alpenverein.de/ der-dav/presse/hintergrund-info/klettern-indeutschland-zahlen-daten-fakten_aid_31813. html.Zugegriffen: 27. Mai 2021.

DOSB (2020). Corona-Schäden für Sportdeutschland 2: Deloitte-Erhebung der finanziellen Implikationen der COVID-19-Pandemie für DOSB-Mitgliedsorganisationen und DOSB-nahe Institutionen, Deutscher Olympischer Sportbund. https://cdn. dosb.de/user_upload/www.dosb.de/Corona/ Coronabedingter-Schaden_Zweite_DeloitteBefragung.pdf.Zugegriffen:25. Apr. 2021.

Eikenbusch, G. (2019). Jugendliche in den Sekundarstufen. Entwicklungsprozesse, Lebenslagen, pädagogische Perspektiven (Pädagogik). Weinheim, Basel, München: Beltz. https://contentselect.com/de/portal/media/view/5c84e9c99ad8-46bf-80fc-646eb0dd2d03?forceauth $=1$. Zugegriffen: 26. Mai 2021.

Kletterhallenverband (KLEVER) e. V. (2021). Mitglieder. https://dkhv.de/mitglieder.Zugegriffen:25. Apr. 2021.

Kohake, K., \& Richartz, A. (2020). „Am meisten macht es immer Spaß, wenn man was Neues schafft" - Das Bedürfnis nach Kompetenzerleben im Kindersport. Forum Kinder- und Jugendsport, 1(2), 110-121. https://doi.org/10.1007/s43594020-00015-4

Konowalczyk, S. (2016). Zeitperspektiven von Jugendlichen. Bildung und Sport, Bd. 11. Wiesbaden: Springer. https://doi.org/10.1007/978-3-65816929-9.

Langmeyer, A., \& Winklhofer, U. (2014). Taschengeld und Gelderziehung. Eine Expertise zum Thema Kinder und ihr Umgang mit Geld mit aktualisierten Empfehlungen zum Taschengeld. München. https://www.dji.de/veroeffentlichungen/ literatursuche/detailansicht/literatur/20604taschengeld-und-gelderziehung.html. Zugegriffen: 7. Juni 2021. 
Memphis Rox (2021). https://www.memphisrox.org/. Zugegriffen:27. Mai 2021.

Neuber, N. (2007). Entwicklungsförderung im Jugendalter.Schorndorf:Hofmann.

Neuber, N. (2011). Bildungspotentiale im Kinderund Jugendsport - Perspektiven für einen zeitgemäßen Bildungsbegriff. In M. Krüger \& N. Neuber (Hrsg.), Bildung im Sport. Beiträge zu einer zeitgemäßen Bildungsdebatte 1. Aufl. Bildung und Sport, (Bd. 1, S. 143). Wiesbaden:VS https://doi.org/10.1007/978-3-531-94026-7.

Walper, S., Reim, J., Schunke, A., Berngruber, A. \& Alt, P. (2021). Die Situation Jugendlicher in der Corona-Krise. Halle. https:// www.dji.de/fileadmin/user_upload/bibs2021/ 2021-05-21_Walper\%20et\%20al_2021_Die \%20Situation\%20Jugendlicher\%20in\%20der \%20Coronakrise_1205\%20\%28003\%29.pdf. Zugegriffen:25. April 2021. 